\title{
ON ULTRACONNECTED SPACES
}

\author{
P.M. MATHEW \\ Department of Mathematics and Statistics \\ Cochin University of Science and Technology \\ Cochin 682 022, India
}

(Received March 17, 1988 and in revised form March 16, 1989)

ABSTRACT. In this paper, we study some properties of ultraconnected spaces and show that ultraconnected $T_{1}$ spaces are maximal ultraconnected and minimal $T_{1} \cdot$ We also introduce the notion $\frac{1}{2}$ of F-connected spaces, topological spaces which are both hyperconnected and $u 1 t$ raconnected and characterize compact maximal F-connected topologies on a set.

KEY WORDS AND PHRASES. U1traconnected, hyperconnected, Semi-topologica1, generalized closed.

1980 AMS SUBJECT CLASSIFICATION CODES. 6A; 54D, 54G.

1. INTRODUCTION.

A topological space is ultraconnected if the intersection of any two nonempty closed sets is nonempty (Steen and Seebach [1]). Each topology $\tau$ on a set $X$ may be associated with a pre-order relation $\rho(\tau)$ on $X$, defined by $(a, b) \varepsilon \rho(\tau)$ if every open set containing b contains a. In 1978 Andima and Thron [2] defined a topological space $(X, \tau)$ to be upward directed if any two elements in $(X, \rho(\tau)$ ) have an upper bound, and it can easily be seen that the notion of upward directed and that of ultraconnected are equivalent.

Let $(X, R)$ be a pre-ordered set. Define $\bar{x}\}=\{y \varepsilon x \mid x R y\}$ and $\{x\}=\{y \varepsilon x \mid y R x\}$, for each $x \in X$. $\mu(R)$, the point closure topology of $R$, is the smallest topology in which all sets $\{\bar{x}\}, x \in X$, are closed and $V(R)$, the kerne1 topology of $R$, is the topology with basis $\{\hat{x}\} \mid x \varepsilon X\}$. A topology $\tau$ on $X$ induces a pre-order $R$ as described above iff $\mu(R) \subset \tau \subset V(R)[2]$.

2. ULTRACONNECTED SPACES.

In [2], it is proved that a topological space $(X, \tau)$ is maximal upward directed iff $(X, \rho(\tau))$ is a partially ordered set of length 1 , with a greatest element and 
$\tau=V(\rho(\tau))$. If $(X, R)$ is a partially ordered set of length 1 , with a greatest element, say a, then $V(R)=P(X \backslash\{a\}) U\{X\}$. Thus the maximal ultraconnected topologies on a set $X$ are precisely $P(X \backslash\{a\}) \cup\{X\}$, where a $\varepsilon X$. DEFINITION 2.1. A topological space is $T_{\frac{1}{2}}$ if each singleton subset is either open
or closed (Levine [3]).

REMARK 2.1. Any $\mathrm{T}_{1}$ space is $\mathrm{T}_{0}$ and Dunham [4] characterized the minimal

$T_{1}$ topologies on a $\operatorname{set}^{\frac{1}{2}} X$ as those of the form $\{0 \subset x \mid 0 \subset A$ or $A C 0$ and 0 'finite $\}$, for some proper subset $A$ of $X$. (When $X$ is finite with more than one element, $A$ must also be nonempty.) Obviously, any maximal ultraconnected space is minimal $\mathrm{T}_{1}$.

THEOREM 2.1. Any ultraconnected $\mathrm{T}_{\frac{1}{2}}$ space is maximal ultraconnected and minima1 $\mathrm{T}_{1} \quad \frac{1}{2}$

PROOF. Let $(X, \tau)$ be an ultraconnected $T_{\frac{1}{2}}$ space. Since $(X, \tau)$ is $T_{\frac{1}{2}}$ the induced order $\rho(\tau)$ is a partial order. Suppose there exist $x, y, z \in X$ such that $x^{\overline{2}} \rho(\tau) y$ and $y \rho(\tau) z$. If $\{y\}$ is open, then $x \rho(\tau) y=\Rightarrow x \varepsilon\{y\} ; i . e ., x=y$. On the other hand, if $\{y\}$ is closed, then $y \rho(\tau) z \Rightarrow z \varepsilon\{\bar{y}\}=\{y\}$; i.e., $z=y$. Since the singletons are either open or closed, it is evident that the length of $(X, \rho(\tau))$ is at most 1 .

If $\{x\}$ is open and $y \rho(\tau) x$, then $y=x$ and hence $x$ is minimal in $(x, \rho(\tau))$. Similarly if $\{x\}$ is closed, then $x$ is maximal in $(X, \rho(\tau))$. Since (X, $\tau)$ is ultraconnected any two minimal elements have an upper bound and there exists only one maximal element which will be the greatest element in $(X, \rho(\tau))$. Moreover, if $x$ is minimal in $(X, \rho(\tau))$, then $\{x\}$ is open and not closed. Hence $\tau=V(\rho(\tau))$. Thus $(X, \tau)$ is maximal ultraconnected, and by the above remark it is minimal $T_{1}$ too.

NOTE 2.1. Though every maximal ultraconnected space is minimal $T_{\frac{1}{2}}$, there are minmal $\mathrm{T}_{\frac{1}{2}}$ spaces which are not even ultraconnected. However, every minimal $\mathrm{T}_{\frac{1}{2}}$ space is connected [4].

Let $X$ be a set with 3 or more elements and $\phi \neq A<X$ such that $|X| A \mid>2$. Then $\tau=\left\{0 \subset x \mid 0<A\right.$ or $A \subset 0$ and $0^{\prime}$ finite $\}$ is a minimal $T_{1}$ topology, which is not ultraconnected. For if $x, y \in X \backslash A$, then $\{x\}$ and $\{y\}$ are closed subsets of (X, $\tau$ ) with empty intersection.

DEFINITION 2.2. A subset of a topological space is called ultraconnected if it is ultraconnected as a subspace.

REMARK 2.2. We will call two subsets $A$ and $B$ of a topological space (X, $\tau$ ) equivalent $(A \equiv B)$ if every open set containing $A$ contains $B$ and conversely. $A^{*}=\cap\{0 \varepsilon \tau \mid 0 \supset A\}$ is the largest subset of $X$ equivalent to $A$. Note that, if $A C B C C$ and $A \equiv C$, then $A \equiv B$ and $B \equiv C$.

THEOREM 2.2. Let $A$ and $B$ be subsets of a topological space $(X, \tau)$ and $A \equiv B$. Then A is ultraconnected iff $B$ is ultraconnected. 
PROOF. Suppose A is ultraconnected, but $B$ is not. Then there exist two nonempty disjoint closed sets $C_{1}, C_{2}$ in $B$. Let $C_{i}=D_{1} B ; 1=1,2 ; D_{1}$ closed in $(X, \tau)$.

$$
C_{1} \cap C_{2} \neq \phi \Rightarrow D_{1} \cap D_{2} \cap B=\phi \Rightarrow B C D_{1}^{\prime} \cup D_{2}^{\prime}
$$

Since $A \equiv B, A C D_{1} ' \cup D_{2}^{\prime}$ and hence $D_{1} \cap D_{2} \cap A=\phi$. But $D_{1} \cap A \neq \phi$, for otherwise $A C D_{1}^{\prime} \Rightarrow B C D_{1}^{\prime} \Rightarrow C_{1}=D_{1} \cap B=\phi . \quad$ similarly $D_{2} \cap A \neq \phi . \quad$ since $D_{1} \cap A$, D $D_{2} A$ are nonempty disjoining closed sets in $A$, we get a contradiction. Hence the result.

DEFINITION 2.3. A subset $A$ of a topological space is generalized closed if $\bar{A} \subset 0$ and 0 whenever $A C O$ and 0 is closed [3].

COROLLARY 2.1. If $A$ is a generalized closed subset of $(X, \tau)$, then $A$ is ultraconnected iff $\bar{A}$ is ultraconnected.

PROOF. In view of Theorem 2.2, it is sufficient to show that $A \equiv \bar{A}$. Since $A$ is generalized closed, if $A \subset 0 \in \tau$, then $\bar{A} C 0$. The other implication is trivial.

COROLLARY 2.2. If $A$ and $B$ are subsets of a space $(X, \tau)$ such that $A C B C A^{*}$, then $A$ is ultraconnected iff $B$ is ultraconnected.

PROOF. Since $A \subset B C A^{*}$ and $A \equiv A^{*}$, it follows that $A \equiv B$ (see the previous remark). Thus the conclusion is an immediate consequence of Theorem 2.2.

DEFINITION 2.4. A subset $A$ of a space $X$ is called semi-open if there exists an open set 0 such that $0 \subset A \subset \overline{0}$ (Levine [5]). A semi-homeomorphism is a bijection under which both images and inverse images of sem-open sets are semi-open. A topological property invariant under semi-homeomorphisms is called a semi-topological property by Crossley and Hildebrahd [6].

REMARK 2.3. Ultraconnectedness is not semitopological. Let $X=\{a, b, c\}$. $\tau_{1}=\{\phi,\{a\},\{a, b\}, X\}$ and $\tau_{2}=\{\phi,\{a\},\{a, b\},\{a, c\}, X\}$. Now $\left(X, \tau_{1}\right)$ is ultraconnected, but $\left(X, \tau_{2}\right)$ is not, while $\tau_{1}$ and $\tau_{2}$ yield the same collection of semiopen sets and hence are semi-homeomorphic.

\section{F-CONNECTED SPACES.}

A topological space in which the intersection of any two nonempty open sets is nonempty is called hyperconnected [1]. We define a topological space to be Fconnected if it is both hyperconnected and ultraconnected.

REMARK 3.1. In the above remark $\left(X, \tau_{1}\right)$ is $F$-connected while $\left(X, \tau_{2}\right)$ is not. Hence $F$-connectedness is not a semi-topological property. Neither the foin nor the product of two F-connected topologies on a set are F-connected. Let $\tau_{1}=\{\phi, A, X\}$ and $\tau_{2}=\{\phi, B, X\}$ where $A \cap B=\phi$. Then $\tau_{1} V \tau_{2}$ and $\tau_{1} \times \tau_{2}$ are not $F$-connected but $\tau_{1}$ and $\tau_{2}$ are F-connected.

THEOREM 3.1. Every subspace of a topological space $(X, \tau)$ is F-connected iff $\tau$ is nested.

PROOF. Necessity: Assume $\tau$ is not nested. Then there exist $A, B \subset X$ such that $A \notin B$ and $B \notin A$. Choose $x \in A \backslash B$ and $y \in B \backslash A$. Then the subspace $\{x, y\}$ has the discrete topology which is obviously not F-connected. 
Sufficiency: Let $\tau$ be nested and $A C X$. Let $0_{1}, 0_{2}$ be nonempty open sets in $A$. Then there exist $B_{1}, B_{2} \varepsilon \tau$ such that $O_{1}=A \cap B_{1}$ and $O_{2}=A \cap B_{2}$. Since $\tau$ is nested, $B_{1} \subset B_{2}$ or $B_{2} \subset B_{1}$. Assume $B_{1} \subset B_{2}$. Then $0_{1} \subset 0_{2}$ and hence $0_{1} \cap 0_{2} \neq \phi \cdot$ Similariy, the intersection of any two nonempty closed sets in $A$ is also nonempty. Thus $A$ is Fconnected.

THEOREM 3.2. If $U$ is an ultrafilter on $X \backslash\{$ a $\}$, for some a $\varepsilon X$, then $\tau=\{\phi, X\} \cup U$ is a maximal F-connected topology on $X$.

PROOF. Obviously, $(X, \tau)$ is F-connected. Suppose $\left(X, \tau_{1}\right)$ is $F$-connected and $\tau_{1}>\tau_{0}$ Let $A \in \tau_{1} \backslash \tau$. Since $A \in \tau_{1} \backslash \tau$, a \&A. For if a $\varepsilon A$, then $\{a\} \cap(X \mid A)=\phi$, a contradiction since $\left(X, \tau_{1}\right)$ is ultraconnected. Now a $k A$ and $A \xi U$ implies $(X \backslash\{a\}) \backslash A \varepsilon$. Thus $A$ and $(X \backslash\{a\}) \backslash A$ are two nonempty disjoint open sets in $\left(X, \tau_{1}\right)$, a contradiction. Hence the result.

THEOREM 3.3. Any compact, maximal F-connected topology on a set $X$ is of the form $\tau_{a}=\{\phi, X\} \cup U_{a}$, where $U_{a}$ is an ultrafilter on $X I\{a\}$, for some a $\varepsilon X$.

PROOF. Let $(X, \tau)$ be compact and maximal F-connected. Since the family of all the nonempty closed sets has finite intersection property and $(x, \tau)$ is compact, it has nonempty intersection. Choose a $\varepsilon \cap\{C \subset x \mid C$ is closed and nonempty \}. Thus the proper open sets are subsets of $X \backslash\{a\}$ and they form a filter base $F$. Let $U_{a}$ be an ultrafilter on $X \backslash\{a\}$ containing $F$. Then $\tau \subset\{\phi, X\} \cup U_{a}=\tau_{a} \cdot$ Since $(X, \tau)$ is maximal F-connected in view of Thoerem 3.2, $\tau=\tau_{a}$.

ACKNOWLEDGMENT. The author wishes to thank Professor T. Thrivikraman for his guidance during the preparation of this paper. He also wishes to thank the referee for the valuable comments which improved the presentation considerably.

\section{REFERENCES}

1. STEEN, L.A. and SEEBACH, J.A., Jr., Counter Examples in Topology, Springer Verlag New York, 1978.

2. ANDIMA, S. J. and THRON, W.J., Order-induced Topological Properties, Pacific J. Math. 75 (1978), 297-318.

3. LEVINE, N., Generalized Closed Sets in Topology, Rend. de1. Circ. Mat. Di. Palermo 19 (1970), 89-96.

4. DUNHAM, W., $T_{\frac{1}{2}}$ spaces, Kyumpook Math. J. 17(2) (1977), 161-169.

5. LEVINE, N., Semi-open Sets and Semi-continuity in Topological Spaces, Amer. Math. Month1y 70 (1963), 36-41.

6. CROSSlEY, S.G. and HILDEBRAND, S.K., Semitopologial Properties, Fund. Math. LXXIV (1972), 232-254.

7. NOIRI, T., Functions which Preserve Hyperconnected Spaces, Rev. Roumaine Math. Pures App1. 25 (1980), 1091-1094. 


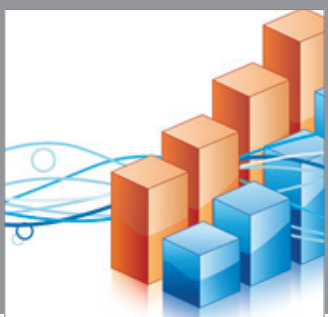

Advances in

Operations Research

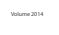

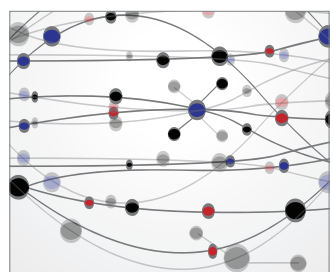

\section{The Scientific} World Journal
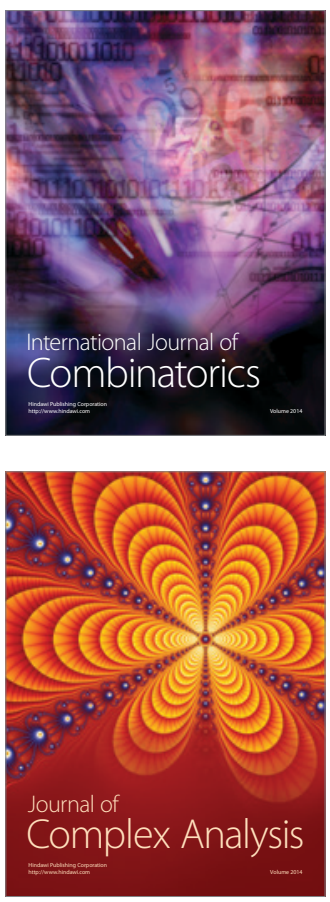

International Journal of

Mathematics and

Mathematical

Sciences
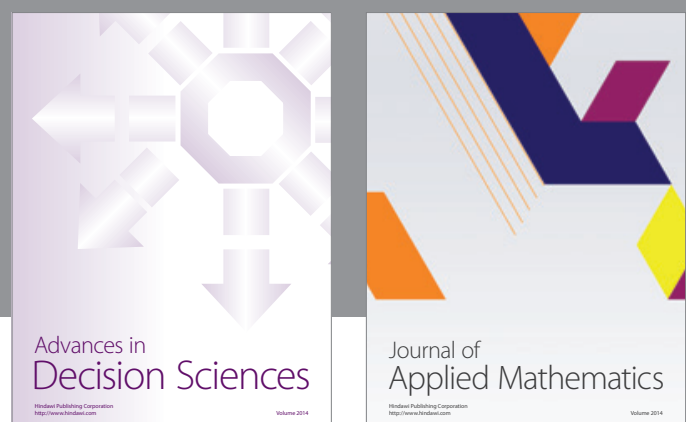

Journal of

Applied Mathematics
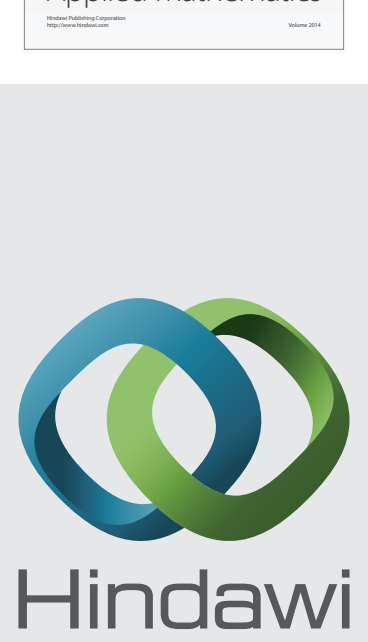

Submit your manuscripts at http://www.hindawi.com
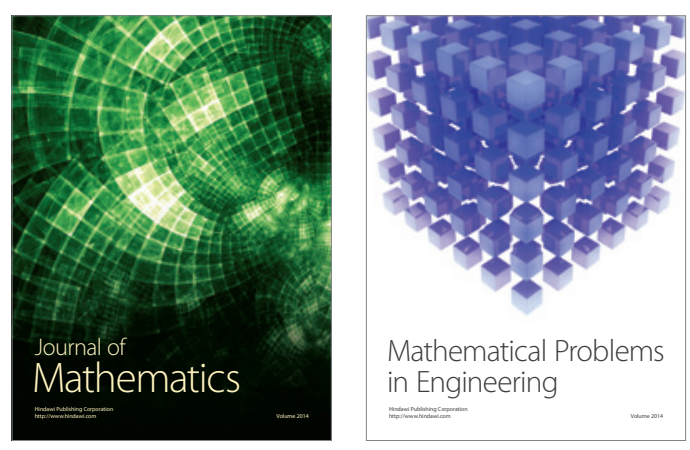

Mathematical Problems in Engineering
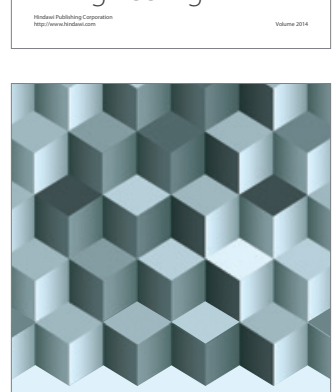

Journal of

Function Spaces
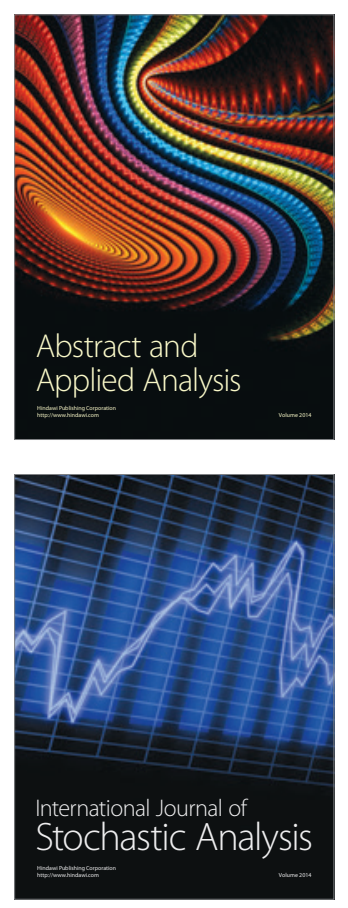

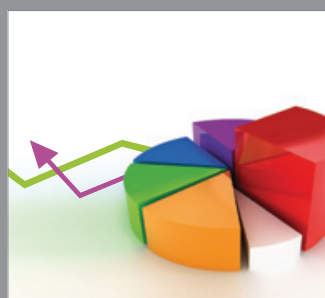

ournal of

Probability and Statistics

Promensencen
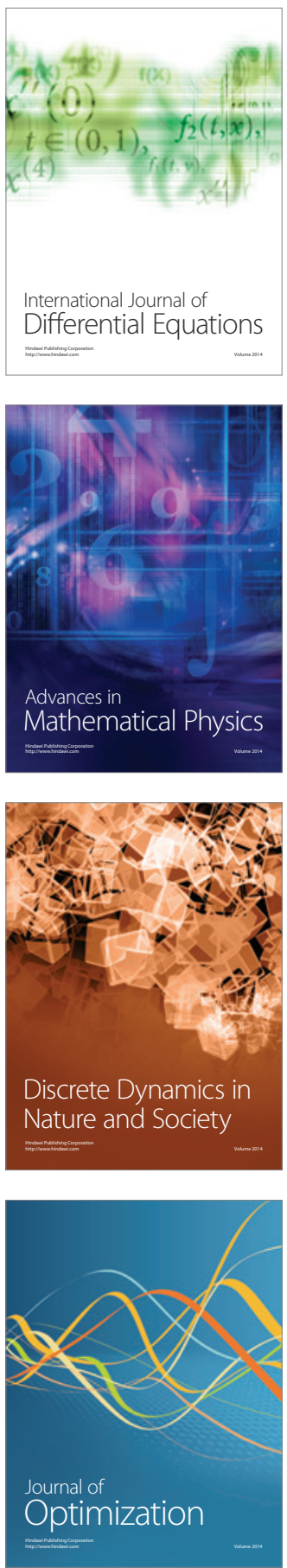